\title{
Responses to Subpar Performance in Foreign Subsidiaries
}

\author{
Vanessa C. Hasse
}

Ph.D. awarded by Western University, Canada (August 2016)

\author{
Big Question: \\ "When foreign subsidiaries experience subpar perfor- \\ mance, what responses can be observed (if any), how ef- \\ fective are these for recovery and survival, and what role \\ does the timing of a response play?"
}

\section{Introduction}

Many countries experienced an extended period of extraordinary growth in prosperity after World War II. This has largely been mirrored by the international business literature where scholars emphasized growth-related themes such as understanding the global business environment of multinational enterprises (MNEs), internationalization, entry mode decisions, international collaborations, and foreign direct investment (Tsui, 2007; Werner, 2002). Meanwhile, the phenomenon of subpar performance has received much less attention, even more so at the level of foreign subsidiaries. Several statistics as well as anecdotal evidence suggest, however, that subpar performance is a reality that warrants a much deeper understanding in order to allow for effective responses with appropriate strategies. Trahms, Ndofor, and Sirmon (2013: 1278), for instance, note that "about half (49.8\%) of firms in the S\&P 500 index for [...] 2010 had experienced more than 3 years of decline within the prior 5 years.", highlighting that the exploration of the subpar performance phenomenon is "likely to remain a highly relevant concern to managers worldwide."

Extant research about the subpar performance phenomenon is somewhat scattered across research realms and mainly attributable to three distinct literatures. First, in the strategic management realm, the organizational decline and turnaround literature (e.g., Trahms et al., 2013) focuses particularly on the phenomenon of subpar performance but relatively neglects the level of foreign subsidiaries, where factors different from the corporate level of analysis may have a significant impact.
For instance, the specific country-level context a subpar-performing foreign subsidiary is embedded in, the role the foreign subsidiary may play within an MNE's network, or the number of expatriates employed at the foreign subsidiary are relevant factors that have not yet received sufficient scholarly attention. Second, in the international strategy realm, the de-internationalization literature (e.g., Benito, 2005; Benito \& Welch, 1997; Berry, 2013) does consider the level of foreign subsidiaries but views subpar performance as one of many motives for the divestment of foreign subsidiaries. It thus lacks depth when it comes to understanding the complexity of responding specifically to subpar performance at the foreign subsidiary level. Third, in the behavioral decision-making realm, the escalation of commitment literature (e.g., Sleesman, Conlon, McNamara $\&$ Miles, 2012) stands out as a framework that emphasizes the psychological mechanisms behind undue persistence in light of subpar performance. While offering valuable insights, this body of literature also relatively neglects the context of foreign subsidiaries while relying to a larger extent on laboratory or classroom samples (Shapira, 1997).

What emerges from this review of the literature is the realization that the fragmentation of research on the subpar performance phenomenon has hampered a holistic understanding of the matter. In fact, some findings appear to be contradictory which renders little assistance to practitioners facing all-toocommon dilemmas such as whether they should "persist and risk becoming caught up in a spiral of escalating commitment, or 'apply the brakes' when they may be within an ace of success" (Drummond, 2014: 430). Further, while some insights exist for addressing subpar performance at the level of the organization overall, there appears to be a paucity of research specifically addressing the phenomenon at the level of foreign subsidiaries. It is thus at the intersection of these literatures where there is a real need for advancing knowledge regarding the selection of appropriate response strategies at the level of subpar performing foreign subsidiaries, including the exploration of specific contingencies. Finally, this dissertation also aims to respond to several calls for a greater degree of integration of 
existing knowledge (e.g., Sleesman et al., 2012; Trahms et al., 2013), encompassing predictors, outcomes, and further theoretically relevant concepts relating to subpar performance such as time. The research in this dissertation is thus guided by three interconnected research questions: When a foreign subsidiary experiences (repeated) subpar performance, what determines which specific type of response is chosen (if any at all)? Which type of response (if any) is most conducive to increasing recovery and survival prospects? What factors determine the timing of a response and what role does the timing of responses play in the effectiveness of the chosen response in increasing recovery and survival prospects?

\section{Overview of the Dissertation Research}

Given the important role resources play during a situation of subpar performance and the facilitation of a turnaround, this dissertation mainly draws from the tradition of the resource-based view (Wernerfelt, 1984; Barney, 1991), its extrapolations in the direction of the resource orchestration framework (Sirmon, Hitt, Ireland \& Gilbert, 2011), and additional tenets from the attention-based view and related constructs. By way of dimensionalizing the phenomenon according to the three research questions, I introduce a framework that integrates the 1) identification of response determinants ("identifying"), 2) assessment of response effectiveness ("responding"), and 3) exploration of the predictors and outcomes of response timing ("synchronizing"). The sample for the analysis was drawn from a large dataset of Japanese worldwide investments (Toyo Keizai, 2014), covering 94 countries over a timeframe from 1990 to 2013. General descriptive findings revealed that the subpar performance phenomenon is indeed quite prevalent, with many subsidiaries in the sample experiencing as much as ten or more consecutive years of subpar performance. Remarkably, the most frequent sequences were those in which no response to the subpar performance could be detected, at least according to the responses measured in the dissertation research.

In the first part of the proposed framework ("identifying"), I contend that the determinants of a response to subpar performance at a foreign subsidiary may reside at different levels of analysis. Indications of favorable country-level conditions revealed to be a significant determinant of an increase in commitment to the subpar-performing foreign subsidiary, while subpar performance at the overall MNE-level was associated with a higher likelihood of inaction as a "response" to the subpar performance at the foreign subsidiary. At the subsidiary-level, a larger number of expatriates was indicative of a higher likelihood of receiving a response, a specific function of the subsidiary (regional headquarters, $R \& D$ center) reduced the likelihood of a decrease in commitment, and subsidiary age was found to increase the likelihood of a decrease in commitment. Finally, if the foreign subsidiary had more than one parent firm, it was more likely to experience a combination of different types of responses.

In the second part of the framework ("responding"), support was garnered for the notion that a response was generally more conducive to the long-term survival prospects of the foreign subsidiary than a non-response. In the short-term, responses may be followed by adjustment periods which may prolong the subpar performance period. Moreover, increases in commitment appeared to often have a more beneficial effect than decreases in commitment.

Finally, in the third part of the framework ("synchronizing"), the existence of communication channels that direct headquarter attention were found to improve the timeliness of a response. More specifically, when the subsidiary had an R\&D focus or the general manager of the foreign subsidiary was Japanese (i.e. the nationality of the focal headquarters), the time to a response was reduced. Further, the relationship between the time-to-first-response and the probability of recovery (versus exit) was concave (inverted U-shape), such that recovery is most likely when the response occurs at a medium amount of time (3 to 6 years) after the onset of the subpar performance sequence. This curvilinear relationship was amplified for deceases in commitment, suggesting that the effectiveness of such responses is more sensitive to timing than increases in commitment. Finally, results suggested that only very early replacements of general managers enhance the likelihood of recovery while later replacements may be counterproductive to turnaround efforts.

\section{Implications for Scholars and Practitioners}

The overarching motivation for this dissertation was to pursue a more differentiated understanding of the complexity of the subpar performance phenomenon by shifting attention to the level of foreign subsidiaries. Within this premise, the objective was to facilitate theoretical development by introducing a framework, thereby helping to bridge the gap in the literature that resulted from a lack of integration. Several contributions for scholars and practitioners emerge.

For scholars, the framework proposed in this dissertation offers a means to organize the complexity of the phenomenon in a way that facilitates subsequent investigations into the matter, underscoring this dissertation's objective to serve as a starting point for the exploration of a number of research directions. Moreover, shifting the perspective to the level of foreign subsidiaries opens up a plethora of new avenues that may previously have been overlooked when focusing on the level of domestic 
operations and/or organizations as a whole. For instance, the impact of different types of distances, cultural differences, or subsidiary network effects materialize as relevant but hitherto largely underexplored factors in examining the phenomenon of subpar performance and could be explored further at this level of analysis. The dissertation research also allows for a quantification of the phenomenon and thus an acknowledgement of it being a prevalent phenomenon to study - not just from the angle of theoretical interest but also practical relevance.

More specifically pertaining to each of the three distinct literatures that are tangential to the phenomenon of subpar performance in foreign subsidiaries, this dissertation aims to inform each in the following main ways. Regarding the organizational decline and turnaround literature, besides a relative neglecting of the foreign subsidiary level, the normative nature of the research has also led to many recommendations pointing in the direction of retrenchment (especially following Pearce and Robbins' work, e.g., Pearce \& Robbins, 1993). The insights gathered in this dissertation, however, suggest that given certain conditions, increases in resource commitment may be preferable instead. Next, the dissertation work is intended to add to the de-internationalization literature by offering a deeper understanding of the determinants of foreign subsidiary divestment under the specific circumstances of subpar performance. Finally, findings from the dissertation contribute to the behavioral decision-makinglescalation of commitment literature by shifting attention to the foreign subsidiary level and suggesting mechanisms that might explain the choices for specific responses.

Beyond these contributions, the dissertation also sheds light on the role that time plays in the context of responding to subpar performance. Many insights in strategic management research rely on assumptions of static conditions, while the longitudinal nature of the dataset used in this dissertation allows for an explicit investigation into the influence of this important moderator and indeed, complex relationships were revealed that future research can build on.

For practitioners, the dissertation offers a structured backdrop for a systematic analysis of a subpar performance situation. For instance, in determining which response to administer, practitioners may consider factors at the country level, MNE-level, and subsidiary level for a more holistic perspective on the complex mechanisms behind the phenomenon. Moreover, the insights gathered in this dissertation suggest that conventional responses such as retrenchment or general manager replacement may not work as universal measures but only under certain conditions. Further, subpar performance situations often constitute high-pressure environments which may prompt "knee-jerk" reactions (Hofer, 1980: 31). The findings from this dissertation indicate, however, that immediate responses may not always be most beneficial to turnaround success. Finally, managerial attention is highlighted as a key resource whose allocation requires deliberate strategic decision-making in order to effectively manage critical situations involving subpar performance. Subpar performance at foreign subsidiaries is different from subpar performance at domestic subsidiaries or the organization overall in the sense that more effort may be required to detect the subpar performance swiftly, identify its likely causes, and administer the necessary response in the timeframe most conducive to the intended outcome. Thus, the careful and deliberate allocation of managerial attention to subpar performance in foreign subsidiaries is an essential organizational task.

In conclusion, a key objective for this dissertation was to inspire more studies on the analysis of the phenomenon of subpar performance in foreign subsidiaries. The proposed framework and findings gathered from a large longitudinal dataset can serve as a stepping stone for future insights in an exciting area of research, thereby starting the next chapter in the exploration of declining organizations and appropriate turnaround strategies. After all, "what we do not know [...] far outweighs what is known about decline and turnaround" (Trahms et al., 2013: 1297).

\section{References}

Barney, J. 1991. Firm resources and stained competitive advantage. Journal of Management, 17(1): 99-120.

Benito, G. R. G. 2005. Divestment and international business strategy. Journal of Economic Geography, 5(2): 235-251.

Benito, G. R. G., \& Welch, L. S. 1997. De-internationalization. Management International Review, 2(Special Issue): 7-25.

Berry, H. 2013. When do firms divest foreign operations? Organization Science, 24(1): 246-261.

Drummond, H. 2014. Escalation of commitment: When to stay the course? Academy of Management Perspective, 28(4): 430-446.

Hofer, C. W. 1980. Turnaround strategies. Journal of Business Strategy. $1(1): 19-30$.

Pearce II, J. A., \& Robbins, K. 1993. Toward improved theory and research on business turnaround. Journal of Management, 19(3): 613-636.

Shapira, Z. B. 1997. Introduction and overview. In Z. B. Shapira (Ed.), Organizational decision making: 3-8. New York: Cambridge University Press.

Sirmon, D. G., Hitt, M. A., Ireland, R. D., \& Gilbert, B. A. 2011. Resource orchestration to create competitive advantage: Breadth, depth, and life cycle effects. Journal of Management, 37(5): 13901412 .

Sleesman, D. J., Conlon, D. E., McNamara, G. \& Miles, J. E. 2012. Cleaning up the big muddy: A meta-analytic review of the determinants of escalation of commitment. Academy of Management Journal, 55(3): 541-562. 
Toyo Keizai. 2014. Kaigai Shinshutsu Kigyo Souran-kuni Betsu. Tokyo: Toyo Keizai.

Trahms, C. A., Ndofor, H. A., \& Sirmon, D. G. 2013. Organizational decline and turnaround: A review and agenda for future research. Journal of Management, 39(5): 1277-1307.

Tsui, A. S. 2007. From homogenization to pluralism: International management research in the academy and beyond. Academy of Management Journal, 50(6): 1353-1364.

Werner, S. 2002. Recent developments in international management research: A review of 20 top management journals. Journal of Management, 28(3): 277-305.

Wernerfelt, B. 1984. A resource-based view of the firm. Strategic Management Journal, 5(2): 171-180.

Vanessa C. Hasse (vhasse@usfca.edu) is an Assistant Professor of International Business at the University of San Francisco. Her research focuses on multinational enterprise strategies, with particular focus on subpar performance at foreign subsidiaries, the complex implications of cultural influences, and other factors impacting subsidiary trajectories. 